\title{
Lesiones Inadvertidas en Trauma Abdominal. Análisis del Trauma Abierto y Cerrado. 12 Casos en el Periodo 1999-2008
}

\author{
Pablo Valsangiácomo, Luis Ruso
}

\section{RESUMEN}

Antecedentes: Analizar los factores determinantes de morbilidad y mortalidad en una serie de pacientes con LI (lesiones inadvertidas) clínicamente relevantes.

Diseño del estudio: E studio retrospectivo, periodo 1999-2008, Hospital Maciel, Montevideo, Uruguay. Las variables analizadas fueron: Edad, sexo, mecanismo lesional, topografía, tiempo de retardo diagnostico, complicaciones, órganos comprometidos y mortalidad.

Se realiza un análisis comparativo en el trauma abierto y contuso.

Resultados: Se reportaron 12 pacientes, todos del sexo masculino, edad media de 29 años con un tiempo de retardo diagnostico de 5,6 días para trauma contuso y de 3,5 para el penetrante. Los órganos comprometidos con mayor frecuencia fueron duodeno, páncreas y colon. Se asociaron mayor demora diagnostica y mortalidad para el trauma contuso de acuerdo al análisis estadístico utilizando el Test exacto de Fisher.

Conclusiones: En retardo diagnostico, el trauma con alta transferencia de energía así como la inadecuada exploración quirúrgica son los factores determinantes en la aparición de complicaciones y mortalidad en lesiones inadvertidas.

Palabras clave: Lesiones inadvertidas, Trauma contuso, Trauma penetrante.

\section{ABSTRACT}

Background: Analyze the determining factors of morbility and mortality in a series of patients with clinically relevant in missed injuries.

Study design: Retrospective study, during the period of 1999 to 2008, Maciel Hospital, Montevideo, Uruguay. The analyzed variables were: age, gender, injury mechanism, topography, time of diagnostic delay, complications, compromised organs and mortality. We undertook a comparative study of penetrating versus blunt trauma.

Results: Twelve patients were reported, all males, mean age of 29 years with a time of diagnostic delay of 5.6 days for blunt trauma and 3.5 for penetrating trauma. The organs that were mainly compromised were the duodenum, pancreas and colon. An increased frequency in diagnostic delay and mortality was associated with blunt trauma according to the Fischer's exact test.

Conclusion: The delay in the diagnosis, the high energy impact injuries, as well as the inadequate surgical exploration are the determining factors for the presence of complications and mortality due to in adverted injuries.

Keywords: In advert injuries, Blunt trauma, Penetrating trauma.

How to cite this article: Valsangiácomo P, Ruso L. Lesiones Inadvertidas en Trauma Abdominal. Análisis del Trauma Abierto y Cerrado. 12 Casos en el Periodo 1999-2008. Panam J Trauma Critical Care Emerg Surg 2012; 1(2):92-94.

\section{Source of support: Nil}

Conflict of interest: None declared

\section{INTRODUCCIÓN}

L a incidencia de lesiones inadvertidas en politraumatizados varía entre 0,5-38\%, variabilidad que se explica según el criterio de inclusión, la definición de LI y el tipo de estudio realizado. ${ }^{1}$ En el trauma abdominal la incidencia de L esiones inadvertidas oscila entre $2-12 \%$. $^{2}$

El objetivo del presente trabajo consiste en analizar las causas y consecuencias del retraso diagnóstico en lesiones abdominal es no diagnosticadas durante la revisión primaria ni secundaria en una serie de pacientes que presentaron trauma abdominal.

Definimos lesión inadvertida a toda lesión traumática luego de realizar la evaluación diagnostica y terapéutica inicial.

\section{MÉTODOS DE INVESTIGACIÓN}

Estudio retrospectivo y observacional durante el periodo 1999-2010 en Hospital Maciel, Montevideo, U ruguay. Las variables analizadas fueron edad, sexo, mecanismo lesional, órganos comprometidos, retraso diagnóstico, complicaciones, mortalidad, realizando un análisis comparativo en trauma abierto y cerrado.

Fueron incluidos pacientes que presentaban trauma abdominal con lesión inadvertida "clínicamente rel evante", definida como aquella que ocasiona morbi mortalidad y que obliga a adoptar una conducta quirúrgica. ${ }^{3}$

La atención primaria del politraumatizado se realiza siguiendo el Advanced Trauma Life Support (ATLS), del A merican College of Surgeons (ACS).

La información fue ordenada en una base de datos y los cálculos efectuados en una planilla electrónica utilizando el programa Microsoft Office Excel 2007. El análisis estadístico se realizo mediante el software SPSS versión 19 para Windows, (SPSS, Chicago IL).

Se consideró estadísticamente significativo $p<0.05$ utilizando el test exacto de Fisher. 


\begin{tabular}{|c|c|c|c|}
\hline \multicolumn{2}{|c|}{ Tabla 1: Trauma contuso } & \multicolumn{2}{|c|}{ Tabla 2: Trauma penetrante } \\
\hline Variables & Media / DE / IC 95\% & Variables & Media / DE / IC 95\% \\
\hline E dad (años) & $33 / \pm 13,6 / 23-43$ & Edad (años) & $28 / \pm 6,5 / 23,2-32,8$ \\
\hline Sexo & M & Sexo & M \\
\hline $\begin{array}{l}\text { Tiempo diagnostico } \\
\text { (días) }\end{array}$ & $5,6 / \pm 4,0 / 2,1-9,1$ & $\begin{array}{l}\text { Tiempo al diagnostico } \\
\text { (días) }\end{array}$ & $3,5 / \pm 3 / 1,3-5,7$ \\
\hline Tipo trauma & $\begin{array}{l}\text { TA - } 3 \\
\text { ABD - } 2 \\
\text { PTM - } 2\end{array}$ & Tipo trauma & $\begin{array}{l}A F-4 \\
A-2 \\
A B-1\end{array}$ \\
\hline Mecanismo de trauma & $\begin{array}{l}A-4 \\
C-3\end{array}$ & Topografía & $\begin{array}{l}\text { TA - } 5 \\
E-1 \\
F-1\end{array}$ \\
\hline Error C/I/Q & $\begin{array}{l}\mathrm{Cl}-\mathbf{4} \\
\mathrm{I}-2 \\
Q-1\end{array}$ & Error C/I/Q & $\begin{array}{l}C-1 \\
Q-4\end{array}$ \\
\hline Órgano lesionado & $\begin{array}{l}\text { Páncreas - } 2 \\
\text { Duodeno - } \mathbf{2} \\
\text { Yeyuno - } 1 \\
\text { Bazo - } 1 \\
\text { Diafragma - } 1\end{array}$ & Órgano lesionado & $\begin{array}{l}\text { Colon - } 4 \\
\text { Duodeno - } \mathbf{3} \\
\text { Páncreas - } 1 \\
\text { Diafragma - } 1 \\
\text { Hígado - } 1\end{array}$ \\
\hline
\end{tabular}

DE: Desvío estándar; IC: Intervalo de confianza; M: Masculino; TA: Toracoabdominal; ABD: Abdomen; PTM: Politraumatizado; A: Aplastamiento; C: Colisión; Cl: Clínico; I: Imagenologico; Q: Quirúrgico; AF: Arma de fuego; AB: Arma blanca

\section{RESULTADOS}

En el periodo analizado se reportaron 12 pacientes que presentaron lesiones inadvertidas abdominales.

Todos los pacientes correspondieron al sexo masculino, edad media de 29 años, DE \pm 8 años, una mediana y una moda de 27 años. El Intervalo de confianza 95\% para la edad fue de 25-33 años. El tiempo de retardo diagnostico fue de 5,6 días para el trauma contuso y 3,5 días para el abierto.

EI mecanismo lesional se repartió en iguales porcentajes para el trauma abierto y cerrado. En el trauma abierto la herida por arma de fuego representó el $66 \%$. L os órganos comprometidos para el trauma contuso fueron duodeno y páncreas mientras que para el trauma abierto fueron duodeno y colon (Table 1 y 2 ).

El $66 \%$ de los casos presentaron complicaciones, destacándose las infecciosas. La mortalidad de la serie fue del $30 \%$.

Realizamos el cálculo de la probabilidad exacta de Fisher, relacionando el tiempo diagnostico, complicaciones y mortalidad en función del trauma abierto o cerrado.

De acuerdo a los resultados podemos inferir que no existe asociación entre el tiempo de demora diagnostica, complicaciones, mortalidad y el tipo de trauma. Tabla 3.

\begin{tabular}{|c|c|c|c|}
\hline & $\begin{array}{l}\text { Trauma } \\
\text { abierto }\end{array}$ & $\begin{array}{l}\text { Trauma } \\
\text { cerrado }\end{array}$ & $\mathrm{p}$ ( $\mathrm{T}$ F isher) \\
\hline Diagnóstico (días) SV/M & $4 / 3$ & $3 / 10$ & 0,173 \\
\hline Complicaciones & $5 / 6$ & $4 / 6$ & 0,500 \\
\hline Mortalidad & $1 / 6$ & $3 / 6$ & 0,545 \\
\hline
\end{tabular}

\section{DISCUSIÓN}

Las lesiones inadvertidas en el paciente politraumatizado son una realidad independiente de la revisión sistemática que se utilice en evaluación inicial y durante la terapéutica. EI no detectarlas a tiempo determina un aumento de la morbimortalidad, mayores costos, repercusiones legales, considerándose un factor de calidad asistencial en servicios de emergencias.

La incidencia de lesiones inadvertidas clínicamente relevantes oscila entre $10-35 \% .{ }^{4}$

Existen múltiples factores que determinan la elevada incidencia de lesiones inadvertidas en trauma, dentro de ellos se destaca el aumento de pacientes en los que se indica el manejo no operatorio (contuso/penetrante) sin reunir todos los criterios necesarios para realizar el mismo.

En nuestra serie en el trauma contuso predomino el error clínico e imagen lógico, mientras que para el trauma penetrante el error ocurrió en la exploración quirúrgica. Figura 1 y 2.

L a sensibilidad de la clínica en el diagnostico de lesiones gastrointestinales en el politraumatizado es del $40 \% .^{5}$

La sensibilidad de la tomografía computada para el diagnostico de lesiones gastrointestinales en el politraumatizado es del $69-97 \%$ y una especificidad del $78-99 \%{ }^{6}$

La evaluación terciaria, concepto introducido por Enderson ${ }^{7}$ implica la reevaluación clínica e imagenológica en las primeras 24 horas, la misma está destinada a minimizar aquellas lesiones inadvertidas en la evaluación primaria y secundaria. 


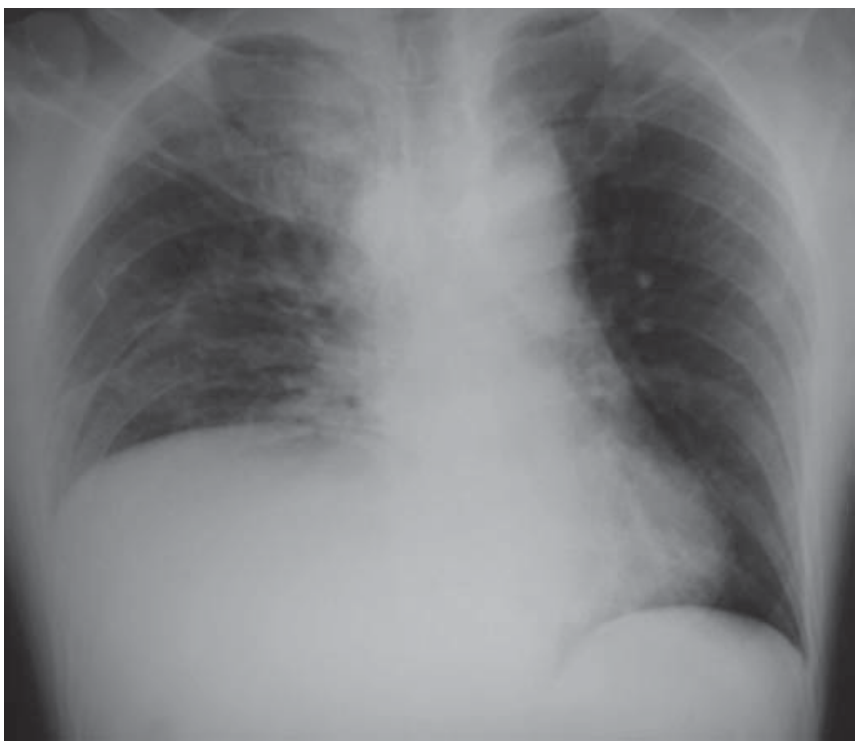

Figura 1: Radiografia de tórax que evidencia ascenso diafragmático derecho. A las 72 hs se realizo diagnostico de hernia diafragmática

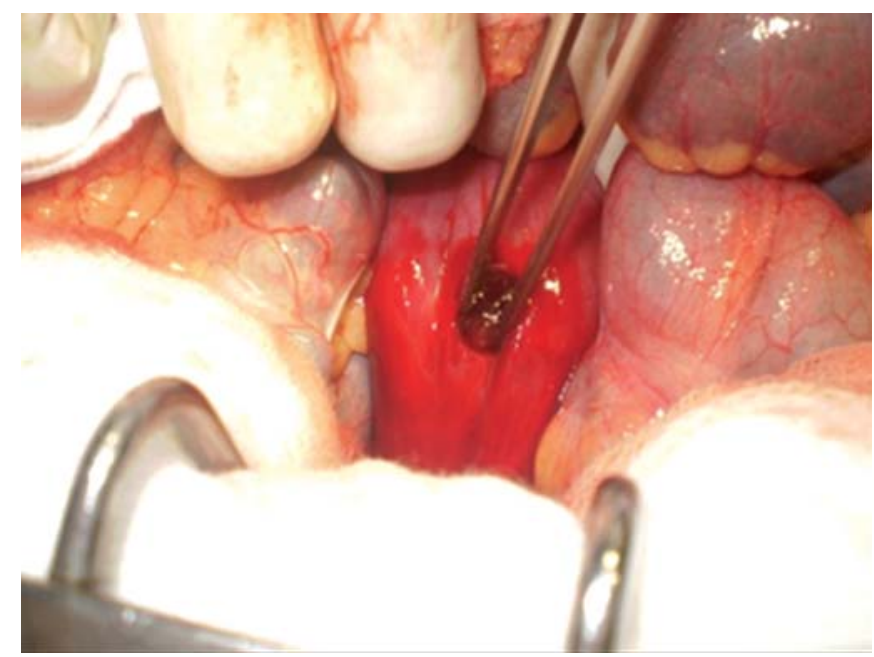

Figura 2: Herida de cara anterior de colon derecho con lesión inadvertida de cara posterior

Existen pacientes de riesgo para presentar lesiones inadvertidas, en nuestra serie el trauma con alta transferencia de energía así como la topografía toracoabdominal, así como la no interpretación adecuada de la imagenología. Otros factores de riesgo mencionados en la literatura son: pacientes con escala de coma de Glasgow menor a 8, inestabilidad hemodinámica, shock neurogénico, índice de severidad lesional mayores a $15 .^{8}$

En la serie analizada no existió diferencia significativa entre el trauma abierto y contuso según el tiempo de demora diagnostica, complicaciones y mortalidad.
L a necesidad de asistencia de los politraumatizados en un centro de trauma, con un equipo multidisciplinario entrenado en trauma así como el seguimiento por el mismo equipo asistencial son factores determinantes en la disminución de la incidencia de lesiones inadvertidas.

Podemos concluir que la no sistematización durante el proceso diagnóstico y terapéutico en el politraumatizado es determinante en la aparición de lesiones inadvertidas. EI retraso diagnóstico así como el inicio tardío de la terapéutica son los principales factores que explican la el evada morbomortalidad de esta situación clínica.

\section{REFERENCIAS}

1. Jiménez-Gomez LM, A munategui I, Sanchez JM, Colón A, Dolores Pérez $M$, Sanz $M$, et al. Lesiones inadvertidas en el politraumatizado: A nálisis de un registro de trauma. Cir Esp 2005;78:303-07.

2. Sung $C K$, Hong K. M issed injuries in abdominal trauma. J Trauma 1996:41:276-78.

3. M ontmany $S, N$ avarro $S$, Rebasa $P$, Hermoso J, Hidalgo J $M$, Cánovas $G$. Estudio prospectivo de la incidencia de las lesiones inadvertidas en el paciente politraumatizada. Cir Esp 2008;84: 32-36.

4. Houshian S, Larsen M, Holm C. M issed injuries in a level I trauma center. J Trauma 2002;52:715-19.

5. Hughes TM D, Elton C, Hitos K, Perez JV, M cDougall PA. Intra-abdominal gastrointestinal tract injuries following blunt trauma: The experience of an A ustralian trauma centre. Injury 2002;33:617-26

6. Valcarcel RC, Turégano FF, Gatica JC, Ruiz de la Hermosa A, Jimenez VW, Diaz PD, et al. Lesiones gastrointestinales y mesenteicas en el paciente politraumatizado: Incidencia, demora diagnostica y pronostico. Cir Esp 2009; 86:17-23.

7. Enderson BL, Reath DB, M eadors J, Dallas W, D eB oo J M , M aull $\mathrm{KL}$. The terciary trauma survey: A prospective study of missed injury. J Trauma 1990;30:666-90.

8. Buduhan G, MCRitchie DI. M issed injuries in patients with multiple trauma. J Trauma 2000;49:600-05.

\section{ACERCA DE LOS AUTORES}

\section{Pablo Valsangiácomo (Correspondencia)}

Ex A sistente de Clínica Quirúrgica, Clínica Quirúrgica '3'. H ospital M aciel, U niversidad de la Republica. Facultad de M edicina, H ospital M aciel, M ontevideo, Uruguay, e-mail: pvalsangiacomo@yahoo.es

\section{Luis Ruso}

Profesor de Clínica Quirúrgica, Clínica Quirúrgica '3'. Hospital M aciel, U niversidad de la Republica. Facultad de M edicina, H ospital Maciel, M ontevideo, U ruguay 